\title{
Enhanced erythrocyte antioxidant status following an 8-week aerobic exercise training program in heavy drinkers
}

Kalliopi Georgakouli ${ }^{a, b}$, Eirini Manthou ${ }^{a, b}$, Ioannis G. Fatouros ${ }^{a, b,}$ Panagiotis Georgoulias ${ }^{c}$, Chariklia K. Deli $^{\mathrm{a}, \mathrm{b}}$, Yiannis Koutedakis ${ }^{\mathrm{a}, \mathrm{b}}$, Yannis Theodorakis ${ }^{\mathrm{a}}$, Athanasios Z. Jamurtas ${ }^{\mathrm{ab}, *}$

\begin{abstract}
Alcohol-induced oxidative stress is involved in the development and progression of various pathological conditions and diseases. On the other hand, exercise training has been shown to improve redox status, thus attenuating oxidative stress-associated disease processes. The purpose of the present study was to evaluate the effect of an exercise training program that has been previously reported to decrease alcohol consumption on blood redox status in heavy drinkers. In a non-randomized within-subject design, 11 sedentary, heavily drinking men (age: $30.3 \pm 3.5$ years; BMI: $28.4 \pm 0.86 \mathrm{~kg} / \mathrm{m} 2$ ) participated first in a control condition for 4 weeks, and then in an intervention where they completed an 8-week supervised aerobic training program of moderate intensity (50e60\% of the heart rate reserve). Blood samples were collected in the control condition (pre-, post-control) as well as before, during (week 4 of the training program), and after intervention (week 8 of the training program). Samples were analyzed for total antioxidant capacity (TAC), thiobarbituric acid reactive substances (TBARS), protein carbonyls $(P C)$, uric acid (UA), bilirubin, reduced glutathione (GSH), and catalase activity. No significant change in indices of redox status in the pre- and post-control was observed. Catalase activity increased $(p<0.05)$ after 8 weeks of intervention compared to week 4 . GSH increased $(p<0.05)$ after 8 weeks of intervention compared to the control condition and to week 4 of intervention. TAC, UA, bilirubin, TBARS, and PC did not significantly change at any time point. Moreover, concentrations of GSH, TBARS, and catalase activity negatively correlated with alcohol consumption. In conclusion, an 8-week aerobic training program enhanced erythrocyte antioxidant status in heavy drinkers, indicating that aerobic training may attenuate pathological processes caused by alcohol-induced oxidative stress.
\end{abstract}

Keywords: Alcohol Abuse, Alcohol-use disorders, Free radicals, Oxidative stress

\section{Introduction}

Light to moderate alcohol use is thought to be beneficial for health; however, excessive alcohol consumption is a leading risk factor for disease development (World Health Organization, 2011). Alcohol is among those factors that can produce increased oxidative stress, which is responsible for the development and/or progression of several diseases (Das \& Vasudevan, 2007; Zima \& Kalousova, 2005). Indeed, heavy drinkers may be more susceptible to oxidative stress than individuals who do not drink heavily (Georgakouli et al., 2015; Tseng et al., 2013), and the pathogenesis of alcohol-related diseases has been found to be associated with alcohol-induced oxidative stress (Arteel, 2003; Lieber, 1994; Lumeng \& Crabb, 2001; Tsukamoto \& Lu, 2001; Zima \& Kalousova, 2005). 
Chronic exercise attenuates oxidative stress by increasing anti- oxidant defense mechanisms (Zalavras et al., 2015). Exercise- induced oxidative stress activates redox-sensitive signaling path- ways that stimulate the expression of certain antioxidant enzymes. The increased antioxidant responses to exercise may be involved in the process of exercise-induced adaptation in skeletal muscle (Coffey \& Hawley, 2007; Steinbacher \& Eckl, 2015). Although heavy drinkers may be more susceptible to oxidative stress, acute exercise causes an antioxidant response similar to those of individuals who do not drink heavily (Georgakouli et al., 2015). However, the long- term effects of exercise training have not been examined yet.

Engaging in regular exercise is a means of improving many aspects of health. Moreover, exercise has been proposed as an adjunct strategy in the treatment of alcohol-use disorders (AUDs) (Georgakouli et al., 2017; Giesen, Deimel, \& Bloch, 2015; Manthou et al., 2016; Zschucke, Heinz, \& Stro€hle, 2012). Therefore, exercise training may help heavy drinkers to reduce their alcohol intake and also improve their health status, a possibility that has not been investigated yet.

In the present study, we applied an aerobic exercise protocol in heavy drinkers to test the hypothesis that exercise training improves indices of blood redox status. The goal of this study is to provide for the first time scientific evidence on the beneficial effects of regular exercise on health aspects of heavy drinkers. Finally, we hope that the results of the present study will highlight the importance of including exercise in the treatment of AUDs and will stimulate further investigation in this topic.

\section{Materials and methods}

\section{Subjects}

Inclusion and exclusion criteria have been described elsewhere (Georgakouli et al., 2017). Briefly, all participants were identified as heavy alcohol drinkers by fulfilling at least one of the criteria set by the National Institute on Alcohol Abuse and Alcoholism (NIAAA, 2015) and had a score of at least 8 at the Alcohol Use Disorders Identification Test (AUDIT) (Moussas et al., 2009). Moreover, they were exhibiting low physical activity levels according to the Greek version of the International Physical Activity Questionnaire (IPAQ- Gr) (Papathanasiou et al., 2009).

Before intervention, participants were informed about the study protocol, the associated risks and benefits, and then signed an informed consent form. Finally, 11 (age: $30.3 \pm 3.5$ years; BMI: $28.4 \pm 0.86$ $\mathrm{kg} / \mathrm{m}^{2}$ ) sedentary, heavily drinking men completed an 8-week supervised aerobic exercise program. The procedures were in accordance with the 1975 Declaration of Helsinki and ethics approval was obtained from the Ethics Committee of the University of Thessaly. This trial was registered at ClinicalTrials.gov as NCT02664766 (U.S. National Institutes of Health, 2016).

\section{Experimental design}

In a non-randomized within-subject design, participants first enrolled in a control condition for 4 weeks, and then in an intervention where they completed an 8-week supervised aerobic training program. During intervention, participants attended at least two 30-min exercise training sessions per week under supervision. Exercise mainly involved walking at moderate intensity (50-60\% of Heart Rate 
Reserve), with heart rate (HR) monitored during exercise by short-range telemetry (Polar RC3 GPS HR; Polar Electro Oy; Kempele, Finland). The intensity of the exercise training maintained between 50 and $60 \%$ of HRR. However, the time of exercise increased throughout the training period. Moreover, participants were asked to record their daily alcohol intake during the control and experimental conditions.

Physiological and biochemical measurements were performed under controlled environmental conditions (room temperature $\sim 25^{\circ} \mathrm{C}$, relative humidity $50-60 \%$ ) before the control condition, as well as before, during (at the end of the $4^{\text {th }}$ week), and after intervention (at the end of the $8^{\text {th }}$ week).

\section{Blood collection and handling}

Participants recorded their diet for 2 days before the first blood collection and followed the same diet before each of the subsequent blood samplings. They were also instructed to avoid any strenuous physical activity for at least 2 days before each blood sample collection. All blood samples were collected in the morning (8:00e9:00 a.m.) after an overnight fast and smoking abstinence.

Blood samples were drawn from a forearm vein with participants in a seated position after resting for 10 min. The procedures for plasma, erythrocyte lysate, and serum preparation from whole blood have been described elsewhere (Georgakouli et al., 2015). Plasma was analyzed for total antioxidant capacity (TAC), thiobarbituric acid reactive substances (TBARS), and protein carbonyls (PC). Erythrocyte lysate was analyzed for reduced glutathione (GSH) and catalase activity, as well as hemoglobin in order to determine the concentrations of GSH and catalase activity. Serum was analyzed for uric acid (UA) and bilirubin.

\section{Blood sample analysis}

Each variable was analyzed in duplicate on the same day, after samples had undergone only one freezethaw cycle. The methods used for determination of the tested indices have also been described elsewhere (Georgakouli et al., 2015).

\section{Statistical analysis}

We performed a power analysis with data from a previous study from our group (Fatouros et al., 2004). The resulting minimum required sample size was 11 for a 2-sided type 1 error of 5\%. The Student's t-test for dependent samples was used to examine control condition and pre-training differences, as well as differences in nutrient content between the 2 days of the diet records. One-way repeated-measures ANOVA was conducted to examine the effect of the training program in all study variables ( 3 time points: 0, 4, 8 weeks). Relationships between variables were tested with Pearson's correlation coefficient. Data are presented as mean \pm standard error of the mean (S.E.M.). The level of statistical significance was set at $p<0.05$. The statistical program used was SPSS version 18.0 (SPSS Inc., United States). 


\section{Results}

No change between the control condition and pre-training measurements were observed (data not shown). Table 1 summarizes anthropometric and physiological characteristics at the baseline (week 0 ). Frequency and duration of exercise training increased significantly from $1.27 \pm 0.54$ to $3.36 \pm 0.24$ sessions per week and $19.09 \pm 7.80$ to $136.64 \pm 16.34$ min per week, respectively. Furthermore, body weight decreased at the 8th week of intervention. These data have been presented previously (Georgakouli et al., 2017). Mean values for nutrient content of the 2-day diet records are summarized in Table 2. Paired samples t-test did not reveal any significant difference in nutrient content between the 2 days.

Regarding indices of blood redox status, GSH significantly increased after week 8 of intervention compared to week $0(p=0.031)$ and compared to week $4\left(p \frac{1}{4} 0.000\right)$ (Fig. 1A). Catalase activity significantly increased $(p=0.011)$ after week 8 of intervention compared to week 4 (Fig. 1B). TAC, UA, bilirubin, TBARS, and PC concentrations did not significantly change after 8 weeks of intervention (Figs. 2 and 3 ).

Alcohol consumption negatively correlated with concentrations of TBARS ( $r=-452 ; p=0.008), \mathrm{GSH}(r=-$ $685 ; p=0.000)$ and catalase activity $(r=-354 ; p=0.043)$. No correlation between duration of exercise per week and any of the indices of redox status was found.

\section{Discussion}

The 8-week supervised aerobic training program of moderate intensity (50-60\% of Heart Rate Reserve) resulted in enhanced erythrocyte antioxidant status in heavy drinkers, as evidenced by the elevation of erythrocyte GSH and catalase activity. Moreover, previously published data have shown that this study protocol led to decreased alcohol consumption by $\sim 36 \%$ after 8 weeks of training (Georgakouli et al., 2017). These results provide evidence of the role of aerobic training in alcohol use reduction and attenuation of pathological processes caused by alcohol-induced oxidative stress in individuals with AUDs.

Few previous studies have investigated the effects of various training protocols on physiological parameters in individuals with AUDs (Brown et al., 2014, 2009; Donaghy, 1997; Ermalinski, Hanson, Lubin, Thornby, \& Nahormek, 1997; Gary \& Guthrie, 1972; Murphy, Pagano, \& Marlatt, 1986; Palmer, Vacc, \& Epstein, 1988; Sinyor, Brown, Rostant, \& Seraganian, 1982; Weber, 1984). This is the first study to examine the effects of exercise training on blood redox status in heavy drinkers. Excessive alcohol use results in oxidative stress because it increases ROS production and de- creases antioxidant defenses (Tseng et al., 2013; Zima \& Kalousova, 2005). Alcohol-induced oxidative stress is associated with the pathogenesis of various diseases (Tsukamoto \& Lu, 2001; Zima \& Kalousova, 2005). On the other hand, exercise training improves redox status by increasing antioxidant defenses and/or reducing production of oxidants. Thus, exercise training protects cells against oxidative stress and damage (Powers, Ji, \& Leeuwenburgh, 1999). We hypothesized that exercise training would improve blood redox status in heavy drinkers who had been previously reported to be more susceptible to oxidative stress, compared to individuals that do not exceed the limits of moderate alcohol use (Georgakouli et al., 2015). The results showed that exercise training improved indices of antioxidant status in erythrocytes; however, 
indices that may reflect the overall redox status of the body (as measured in plasma and serum) did not change. GSH is a major endogenous antioxidant. Blood GSH is primarily contained in the erythrocytes and may be an indicator of resting and exercise-induced oxidative stress (Laaksonen et al., 1999). GSH concentrations may be significantly lower in individuals with AUDs compared to healthy controls (Georgakouli et al., 2015; Loguercio et al., 1999) due to their chronic exposure to alcohol. Therefore, finding ways to increase GSH concentrations in these individuals may help them counterbalance oxidative stress. All types of exercise training enhance GSH concentrations by inducing GSH synthesis and increasing GSH regeneration from its oxidized form (GSSG) (Sen \& Packer, 2000). Although GSH concentrations did not increase after acute aerobic exercise of moderate intensity (Georgakouli et al., 2015), 8 weeks of aerobic training was efficient in increasing erythrocyte GSH.

Catalase is one of the three enzymes that convert alcohol to acetaldehyde; however, its role in alcohol metabolism is limited. Nevertheless, catalase is an important cellular antioxidant enzyme that converts hydrogen peroxide $(\mathrm{H} 2 \mathrm{O} 2)$ to oxygen and water. Ex- ercise training increases catalase activity in sedentary healthy men (Ohno, Yahata, Sato, Yamamura, \& Taniguchi, 1988). In the present study, erythrocyte catalase activity increased at week 8 of intervention compared to concentrations at week 4. Therefore, aerobic training may have resulted in improved enzymatic antioxidant defense against free radicals in erythrocytes of heavy drinkers.

Taken together, increased erythrocyte concentrations of GSH and catalase activity following 8 weeks of aerobic training indicate an upregulation of erythrocyte antioxidant defense in heavy drinkers, who may be more prone to oxidative stress. Thus, aerobic exercise may reduce erythrocyte susceptibility to oxidative stress in heavy drinkers. Interestingly, a negative correlation of alcohol consumption with GSH and catalase activity was also observed. Future studies should distinguish the role of exercise training and reduced alcohol use in modulation of redox status in individuals with AUDs.

Although the concentrations of indices of erythrocyte antioxidant status increased after intervention, the concentrations of TAC in plasma, and UA and bilirubin in serum did not significantly change. Plasma and serum are components of blood that may reflect the overall redox status of the body. Therefore, exercise may have influenced changes in antioxidant status of no other organ or tissue than erythrocytes. Additionally, the aforementioned changes in erythrocyte antioxidant status could only be explained by the exercise protocol that included aerobic activities of moderate in- tensity. Exercise training interventions that include muscle- damaging exercise may influence more changes in indices of oxidative stress (Nikolaidis et al., 2012). Longer interventions or different training protocols may cause greater adaptations and, therefore, influence changes in more indices.

In addition, regarding indices of oxidative stress in plasma (TBARS as an index of lipid peroxidation, PC as an index of protein oxidation), no significant difference was detected after intervention. These results also indicate that exercise training may have influenced changes in erythrocytes but not in overall redox status; however, more prolonged exercise training interventions may lead to changes in these indices as well.

Moreover, there was also a negative correlation between alcohol consumption and TBARS concentrations. Alcohol consumption is associated with lipid oxidation. A study showed that reduction in alcohol intake was accompanied by decreased plasma $F(2)$ - isoprostanes in moderate to heavy alcohol drinkers (40e110 g/ day) (Barden et al., 2007). Furthermore, in another study it was found that serum malondialdehyde concentrations significantly decreased after 3 days of withdrawal treatment in 
alcoholics (Bleich et al., 2000). Thus, it is necessary to conduct interventions over longer periods to achieve a better understanding of the effects of chronic exercise in individuals with AUDs.

Taken all together, various factors could have contributed to the enhanced erythrocyte antioxidant status that was observed following the 8-week training program in heavy drinkers. Weight loss could be one of them. Participants were overweight and their body weight decreased (88.85 \pm 3.05 vs. $87.62 \pm$ 3.04) at the 8th week of intervention (previously published data; Georgakouli et al., 2017). Therefore, a negative energy balance was obtained. Energy restriction has been shown to influence changes in oxidative stress and redox status (Gredilla \& Barja, 2005). However, food intake was not monitored throughout the training program in order to conclude whether weight loss was the result of exercise, hypo- caloric diet or both.

Another factor that could have contributed to the presented results is alcohol. Exercise training was accompanied by a significant decrease in alcohol use, which influences redox status. Thus, the enhanced erythrocyte antioxidant status observed could also be the result of exercise, decreased alcohol consumption, or both. A control group of heavy drinkers that would decrease alcohol consumption without participating in exercise training would help interpret the effects of exercise training on redox status properly.

Finally, the lack of a control group and the small sample size are two important limitations of the study. However, although data cannot be interpreted safely, it cannot be overlooked that exercise training resulted in many favorable changes (i.e., body weight, alcohol use, blood redox status) in heavy drinkers.

\section{Conclusions}

An 8-week aerobic exercise program accompanied by reduced alcohol consumption may enhance erythrocyte antioxidant status in heavy drinkers. Increased antioxidant defense against oxidative stress in erythrocytes may result in attenuated pathological processes caused by alcohol-induced oxidative stress. Based on this and previous studies which reported that exercise training improves redox status and reduces alcohol consumption, it seems reasonable to propose that chronic exercise may be beneficial for individuals with AUDs. More studies using different exercise protocols would provide more evidence on what is the optimal protocol for gaining health benefits in individuals with AUDs.

\section{Funding}

This study was co-financed by the European Union [European Social Fund (ESF)] and Greek national funds through the Opera- tional Program 'Education and Lifelong Learning' of the National Strategic Reference Framework-Research Funding Program: THALES. Investing in knowledge society through the ESF.

\section{Disclosure statement}

The authors declare no conflicts of interest. 
Author contributions

A.Z.J. and Y.T. conceived and designed the experiments; K.G. and E.M. performed the experiments; K.G., E.M., P.G., C.K.D., and A.Z.J. analyzed the data; K.G., I.G.F, Y.K., and A.Z.J. wrote the paper.

Acknowledgments

The authors thank Mr. Ioannis Galanis for his assistance with diet analysis.

References

Arteel, G. E. (2003). Oxidants and antioxidants in alcohol-induced liver disease. Gastroenterology, 124, 778e790. https://doi.org/10.1053/gast.2003.50087.

Barden, A., Zilkens, R. R., Croft, K., Mori, T., Burke, V., Beilin, L. J., et al. (2007). A reduction in alcohol consumption is associated with reduced plasma F2- isoprostanes and urinary 20-HETE excretion in men. $\begin{array}{lllll}\text { Free } \quad \text { Radical } \quad \text { Biology } & \text { Medicine, 42, } & 1730 \mathrm{e} 1735 .\end{array}$ https://doi.org/10.1016/j.freeradbiomed.2007.03.004.

Bleich, S., Spilker, K., Kurth, C., Degner, D., Quintela-Schneider, M., Javaheripour, K., et al. (2000). Oxidative stress and an altered methionine metabolism in alcoholism. Neuroscience Letters, 293, $171 \mathrm{e} 174$.

Brown, R. A., Abrantes, A. M., Minami, H., Read, J. P., Marcus, B. H., Jakicic, J. M., et al. (2014). A preliminary, randomized trial of aerobic exercise for alcohol dependence. Journal of Substance Abuse Treatment, 47, 1e9. https://doi.org/10.1016/ j.jsat.2014.02.004.

Brown, R. A., Abrantes, A. M., Read, J. P., Marcus, B. H., Jakicic, J., Strong, D. R., et al. (2009). Aerobic exercise for alcohol recovery: Rationale, program description, and preliminary findings. Behavior Modification, 33, 220e249. https://doi.org/10.1177/0145445508329112.

Coffey, V. G., \& Hawley, J. A. (2007). The molecular bases of training adaptation. Sports Medicine, 37, 737e763.

Das, S. K., \& Vasudevan, D. M. (2007). Alcohol-induced oxidative stress. Life Sciences, 81, 177e187. https://doi.org/10.1016/j.Ifs.2007.05.005.

Donaghy, M. E. (1997). The investigation of exercise as an adjunct to the treatment and rehabilitation of the problem drinker (dissertation). UK: University of Glasgow.

Ermalinski, R., Hanson, P. G., Lubin, B., Thornby, J. I., \& Nahormek, P. A. (1997). Impact of a body-mind treatment component on alcoholic inpatients. Journal of Psychosocial Nursing and Mental Health Services, 35, 39e45. 
Fatouros, I. G., Jamurtas, A. Z., Villiotou, V., Pouliopoulou, S., Fotinakis, P., Taxildaris, K., et al. (2004). Oxidative stress responses in older men during endurance training and detraining. Medicine and Science in Sports and Exercise, 36, $2065 \mathrm{e} 2072$.

Gary, V., \& Guthrie, D. (1972). The effect of jogging on physical fitness and self- concept in hospitalized alcoholics. Quarterly Journal of Studies on Alcohol, 33, $1073 \mathrm{e} 1078$.

Georgakouli, K., Manthou, E., Fatouros, I. G., Deli, C. K., Spandidos, D. A., Tsatsakis, A. M., et al. (2015). Effects of acute exercise on liver function and blood redox status in heavy drinkers. Experimental and Therapeutic Medicine, 10, 2015e2022. https://doi.org/10.3892/etm.2015.2792.

Georgakouli, K., Manthou, E., Georgoulias, P., Ziaka, A., Fatouros, I. G., Mastorakos, G., et al. (2017). Exercise training reduces alcohol consumption but does not affect HPA-axis activity in heavy drinkers. Physiology \& Behavior, 179, 276e283. https://doi.org/10.1016/j.physbeh.2017.07.003.

Giesen, E. S., Deimel, H., \& Bloch, W. (2015). Clinical exercise interventions in alcohol use disorders: A systematic review. Journal of Substance Abuse Treatment, 52, 1 e9. https://doi.org/10.1016/j.jsat.2014.12.001.

Gredilla, R., \& Barja, G. (2005). Minireview: The role of oxidative stress in relation to caloric restriction and longevity. Endocrinology, 146, 3713e3717. https://doi.org/10.1210/en.2005-0378.

Laaksonen, D. E., Atalay, M., Niskanen, L., Uusitupa, M., Hanninen, O., \& Sen, C. K. (1999). Blood glutathione homeostasis as a determinant of resting and exercise- induced oxidative stress in young men. Redox Report, 4, 53e59. https://doi.org/10.1179/135100099101534648.

Lieber, C. S. (1994). Susceptibility to alcohol-related liver injury. Alcohol and Alcoholism - Supplement, 2, $315 \mathrm{e} 326$.

Loguercio, C., Blanco, F. D., De Girolamo, V., Disalvo, D., Nardi, G., Parente, A., et al. (1999). Ethanol consumption, amino acid and glutathione blood levels in patients with and without chronic liver disease. Alcoholism: Clinical and Experi- mental Research, 23, 1780e1784.

Lumeng, L., \& Crabb, D. W. (2001). Alcoholic liver disease. Current Opinion in Gastroenterology, 17, $211 \mathrm{e} 220$.

Manthou, E., Georgakouli, K., Fatouros, I. G., Gianoulakis, C., Theodorakis, Y., \& Jamurtas, A. Z. (2016). Role of exercise in the treatment of alcohol use disorders. Biomedical Reports, 4, 535e545. https://doi.org/10.3892/br.2016.626.

Moussas, G., Dadouti, G., Douzenis, A., Poulis, E., Tzelembis, A., Bratis, D., et al. (2009). The Alcohol Use Disorders Identification Test (AUDIT): Reliability and validity of the Greek version. Annals of General Psychiatry, 8, 11. https://doi.org/10.1186/1744-859x-8-11.

Murphy, T. J., Pagano, R. R., \& Marlatt, G. A. (1986). Lifestyle modification with heavy alcohol drinkers: Effects of aerobic exercise and meditation. Addictive Behaviors, 11, 175e186.

National Institute on Alcohol Abuse and Alcoholism. (2015). Drinking levels defined. Retrieved from http://www.niaaa.nih.gov/alcohol-health/overview-alcohol- consumption/moderate-binge-drinking. 
Nikolaidis, M. G., Kyparos, A., Dipla, K., Zafeiridis, A., Sambanis, M., Grivas, G. V., et al. (2012). Exercise as a model to study redox homeostasis in blood: The effect of protocol and sampling point. Biomarkers, 17, 28e35. https://doi.org/10.3109/1354750x.2011.635805.

Ohno, H., Yahata, T., Sato, Y., Yamamura, K., \& Taniguchi, N. (1988). Physical training and fasting erythrocyte activities of free radical scavenging enzyme systems in sedentary men. European Journal of Applied Physiology and Occupational Physi- ology, 57, 173e176.

Palmer, J., Vacc, N., \& Epstein, J. (1988). Adult inpatient alcoholics: Physical exercise as a treatment intervention. Journal of Studies on Alcohol, 49, 418e421.

Papathanasiou, G., Georgoudis, G., Papandreou, M., Spyropoulos, P., Georgakopoulos, D., Kalfakakou, V., et al. (2009). Reliability measures of the short International Physical Activity Questionnaire (IPAQ) in Greek young adults. Hellenic Journal of Cardiology, 50, 283e294.

Powers, S. K., Ji, L. L., \& Leeuwenburgh, C. (1999). Exercise training-induced alterations in skeletal muscle antioxidant capacity: A brief review. Medicine and Science in Sports and Exercises, 31, 987e997.

Sen, C. K., \& Packer, L. (2000). Thiol homeostasis and supplements in physical exercise. The American Journal of Clinical Nutrition, 72(2 Suppl), 653Se669S. Sinyor, D., Brown, T., Rostant, L., \& Seraganian, P. (1982). The role of a physical fitness program in the treatment of alcoholism. Journal of Studies on Alcohol, 43, 380e386.

Steinbacher, P., \& Eckl, P. (2015). Impact of oxidative stress on exercising skeletal muscle. Biomolecules, 5, 356e377. https://doi.org/10.3390/biom5020356.

Tseng, Y. M., Tsai, S. M., Lin, C. C., Jin, Y. R., Yeh, W. H., Hsiao, J. K., et al. (2013). Oxidative stressrelated enzyme polymorphisms associated with the immunological biomarkers levels in heavy drinkers in Taiwan. Journal of Clinical Laboratory Analysis, 27, 494e503. https://doi.org/10.1002/jcla.21633.

Tsukamoto, H., \& Lu, S. C. (2001). Current concepts in the pathogenesis of alcoholic liver injury. FASEB Journal, 15, 1335e1349.

U.S. National Institutes of Health. http://www.clinicaltrials.gov.

Weber, A. (1984). Running as treatment for hospitalized alcoholics: An experimental approach. Suchtgefahren, 30, 160e167.

World Health Organization. (2011). Causes of death 2008: Data sources and methods. Geneva: WHO.

Zalavras, A., Fatouros, I. G., Deli, C. K., Draganidis, D., Theodorou, A. A., Soulas, D., et al. (2015). Agerelated responses in circulating markers of redox status in healthy adolescents and adults during the course of a training macrocycle. Oxidative Medicine and Cellular Longevity, 2015, 283921. https://doi.org/10.1155/2015/283921.

Zima, T., \& Kalousova, M. (2005). Oxidative stress and signal transduction pathways in alcoholic liver disease. Alcoholism: Clinical and Experimental Research, 29(11 Suppl), 110Se115S.

Zschucke, E., Heinz, A., \& Strohle, A. (2012). Exercise and physical activity in the therapy of substance use disorders. The Scientific World Journal, 2012, 901741. https://doi.org/10.1100/2012/901741. 


\section{Figures' legends}

Fig. 1. GSH (A) and catalase (B) responses in erythrocyte lysate.

*Significant difference $(p<0.05)$ from the baseline (week 0$)$. "Significant difference $(p<0.05)$ from week 4 of ET intervention.

Fig. 2. TAC (A), protein carbonyls (B), and TBARS (C) responses in plasma.

Fig. 3. Bilirubin (A) and uric acid (B) responses in serum. 